\title{
Expression of Pullulanase from Thermus Thermophilus HB8 in Pichia Pastoris and its Characterization
}

\author{
Mounia Akassou ${ }^{1}$, Jean-François Lemay ${ }^{2}$, Patrick Quessy ${ }^{2}$, Denis Groleau ${ }^{1}$ \\ ${ }^{1}$ Department of Chemical and Biotechnological Engineering, Faculty of Engineering, University of Sherbrooke \\ 2500, boul. de l'Université, Sherbrooke J1K 2R1, Quebec, Canada \\ mounia.akassou@usherbrooke.ca; denis.groleau@usherbrooke.ca \\ ${ }^{2}$ National Center of Electrochemistry and Environmental Technologies (CNETE) \\ 2263, Avenue du Collège, Shawinigan G9N 6V8, Quebec, Canada \\ jflemay@cnete.qc.ca; pquessy@cnete.qc.ca
}

\section{Extended Abstract}

Pullulanase is one of the important debranching enzymes. Its type I is used usually in the saccharification step of starch to catalyse the hydrolysis of the $\alpha$-D-1,6 glucosidic linkages in amylopectin.

The objective of this study was to investigate the feasibility of extracellular production of a thermoduric pullulanase from Thermus thermophilus HB8 by Pichia pastoris MutS strains. Firstly, six secretion signal sequences were tested for extracellular pullulanase accumulation. The expression vector containing the alpha-factor -Kex-Ste of Saccharomyces cerevisiae (pD912-AKS-19) was able to lead to some extracellular pullulanase accumulation clearly detected at the $5^{\text {th }}$ day of methanol-driven induction $(0.14 \mathrm{U} / \mathrm{ml})$. However, more than $98 \%$ of the pullulanase was accumulated intracellularly, contrary to expectations. To enhance the excretion of the recombinant pullulanase, natural osmolytes (proline, guanidine, betaine and K-glutamic acid), and Triton X-100, were tested. The production of extracellular pullulanase was increased more than 50 -fold by adding K-glutamic acid at the final concentration of $0.4 \%(\mathrm{w} / \mathrm{v})$ five hours before induction, and by adding Triton X-100 48h after induction. Afterwards, the effect of temperature and $\mathrm{pH}$ during induction on enzyme levels was evaluated. To characterize the recombinant pullulanase, a semi-purification protocol was firstly applied, made up of two steps: thermal treatment and centrifugation. The optimal $\mathrm{pH}$ and temperature of the recombinant pullulanase were, respectively, 6.0 and $70^{\circ} \mathrm{C} .50 \%$ of the pullulanase activity was recovered after incubation at $70^{\circ} \mathrm{C}$ for $60 \mathrm{~min}$. 